\title{
Desflurano: Propriedades Físico-Químicas, Farmacologia e Uso Clínico *
}

\author{
Desflurane: Physicochemical Properties, Pharmacology and \\ Clinical Use
}

Renato Ângelo Saraiva, TSA ${ }^{1}$

\section{RESUMO}

Saraiva RA - Desflurano: Propriedades Físico-Químicas, Farmacologia e Uso Clínico

Justificativa e Objetivos - Seguindo o desenvolvimento da química nuclear com a síntese dos halogenados desde a década de 50 no século passado, vários agentes foram ensaiados clinicamente e alguns tiveram grande aplicação prática. A busca pelo agente ideal continua. Atualmente estão em uso clínico o halotano, enflurano, isoflurano, sevoflurano e desflurano. Todos apresentam vantagens e desvantagens. O desflurano é o mais recente destes agentes. O objetivo deste trabalho é descrever as propriedades físico-químicas e farmacológicas do desflurano e relatar a aplicação clínica obtida com o uso deste novo agente.

Conteúdo - As propriedades físico-químicas e as características farmacocinéticas e farmacodinâmicas são determinantes do uso clínico do desflurano. Tendo ponto de ebulição baixo, volatiliza facilmente nas temperaturas das salas de operação, e sua CAM elevada requer que seja administrado em concentrações altas. Então, é recomendável o uso de fluxo baixo de gás fresco e vaporizador especial para que sua aplicabilidade clínica seja economicamente viável. Além disto, o uso de um agente coadjuvante, como o óxido nitroso, reduz sua CAM e possibilita ser usado em menores concentrações. Sua farmacocinética permite indução e regressão rápida, salientando-se também que tem molécula muito estável, sendo pouquíssimo metabolizado, oferecendo grande tolerabilidade para o organismo humano. Suas repercussões farmacodinâmicas são doses-dependentes, semelhantes aos demais agentes inalatórios potentes.

Conclusões - O desflurano representa uma etapa a mais na evolução para se chegar ao anestésico ideal. Suas propriedades físico-químicas the conferem características farmacocinéticas bastante desejáveis, que propiciam indução (progressão) e regressão rápidas e também metabolização mínima com a mais baixa toxicidade orgânica entre os anestésicos halogenados, e forte estabilidade molecular, inclusive na presença de absorventes de dióxido de carbono. Tomando-se as devidas precauções quanto à vaporização, armazenamento e consumo, o desflurano pode ser usado inclusive em larga escala, sendo economicamente viável.

UNITERMOS: ANESTÉSICOS, Volátil: desflurano

\footnotetext{
* Recebido da (Received from) Rede Sarah de Hospitais do Aparelho Locomotor, Brasília, DF

1. Coordenador de Anestesiologia da Rede Sarah de Hospitais do Apare-
} Iho Locomotor

Apresentado (Submitted) em 24 de abril de 2002

Aceito (Accepted) para publicação em 02 de setembro de 2002

Correspondência para (Mail to):

Dr. Renato Ângelo Saraiva

SMHS Quadra 501 - Conjunto A

70330-150 Brasília, DF

(C) Sociedade Brasileira de Anestesiologia, 2003

\section{SUMMARY}

Saraiva RA - Desflurane: Physicochemical Properties, Pharmacology and Clinical Use

Background and Objectives - Following the development of nuclear chemistry with halogenate synthesis in the 50's of past century, several anesthetics were clinically studied and some of them had wide practical application. The search for the ideal agent continues. Currently, halothane, isoflurane, enflurane, sevoflurane and desflurane are in clinical use. All have advantages and disadvantages. Desflurane is the newest agent. This study aimed at describing physicochemical and pharmacological properties of desflurane and reporting clinical experiences with this agent.

Contents - Physicochemical properties and pharmacokinetic and pharmacodynamic properties of desflurane determine its clinical use. With a low boiling point, it volatizes easily in normal operating room temperatures and its high MAC requires it to be administered in high concentrations. So, the use of low fresh gas flow and special vaporizer is recommended for it to be economically feasible. In addition, the use of coadjuvant anesthetics, such as nitrous oxide, decreases its MAC and allows it to be used in lower concentrations. Its pharmacokinetics provides fast induction and recovery being also worth mentioning that it has a highly stable molecule and is minimally metabolized, thus being well tolerated by the human body. Its pharmacodynamic repercussions are dose-dependent and similar to other potent inhalation anesthetics.

Conclusions - Desflurane is an additional step in the evolution toward the ideal anesthetic agent. Its physicochemical properties give it highly desirable pharmacokinetic characteristics which provide fast induction (progression) and recovery and also minimal metabolic degradation with the lowest organic toxicity among halogenate anesthetics, in addition to strong molecular stability, even in the presence of carbon dioxide absorbents. With special attention regarding vaporization, storage, and consumption, desflurane may be used even in large scale, being economically feasible.

KEY WORDS: ANESTHETICS, Volatile: desflurane

\section{INTRODUÇÃO}

pós o desenvolvimento da química nuclear nas décadas Ade 40 e 50 do século XX, houve uma grande evolução na síntese de anestésicos inalatórios a partir dos hidrocarbonetos. A halogenização destes compostos, que consiste em substituir átomos de hidrogênio por átomos de flúor ou cloro, resulta na formação de substância com propriedades anestésicas.

Vários destes agentes foram ensaiados clinicamente e alguns tiveram grande aplicação na prática diária.

Atualmente estão em uso o halotano, o enflurano, o isoflurano, o sevoflurano e o desflurano. Estes dois últimos foram introduzidos mais recentemente. 
Ainda não existe o agente ideal, aquele que preencha todas as propriedades desejadas sem oferecer nenhum efeito indesejado. Por esta razão, há sempre novos agentes sendo estudados.

Dos halogenados que estão em uso, o halotano continua sendo muito administrado, especialmente em pediatria, apesar de ser disritmogênico e de desenvolver potencialmente toxicidade, devido a sua instabilidade, agravada pela solubilidade elevada, que favorece a maior absorção e conseqüente grande metabolização. O isoflurano não é bem tolerado para indução por inalação e não oferece uma regressão da anestesia muito rápida. Mesmo assim, sendo bem tolerado pelo organismo é provavelmente, nos dia de hoje, o anestésico inalatório mais utilizado. O sevoflurano tem odor agradável e não é disritmogênico. Por isto está sendo muito administrado em anestesia pediátrica especialmente nas induções inalatórias. No entanto, libera muito flúor e, por esta razão, o seu uso em procedimentos de longa duração não é muito recomendado, especialmente em pacientes com insuficiência renal.

O desflurano tem pouca solubilidade no sangue e tecidos orgânicos, sendo por isto rapidamente absorvido e eliminado. Favorece a progressão da concentração alveolar, que logo se aproxima da CAM e, do mesmo modo, ao término da sua administração, permite que a concentração alveolar, quase imediatamente, passe a ser sub-anestésica, propiciando ao paciente emergir da anestesia muito rapidamente. É pouquíssimo metabolizado e a liberação de flúor sérico da sua molécula é insignificante. Isto lhe confere o conceito de agente mais bem tolerado pelo organismo na atualidade sob o ponto de vista de toxicidade. O seu odor pungente limita muito a sua aplicabilidade em indução inalatória. Então, pode-se imaginar que o desflurano ganha espaço como agente para progressão e regressão rápidas e por ter baixíssima toxicidade, mas perde terreno na indução exclusivamente inalatória e na exigência de um bom controle sobre a umidade de cal sodada para evitar a formação de monóxido de carbono.

Certamente o desflurano, embora não represente um agente que esteja muito próximo daquele que pudesse ser chamado de ideal, representa a conquista de mais degraus nesta direção.

O objetivo deste trabalho é descrever as características físico-químicas e farmacológicas do desflurano e também relatar a aplicação clínica obtida com o uso deste novo agente.

\section{PROPRIEDADES FÍSICO-QUÍMICAS}

Aestrutura molecular do desflurano é semelhante a do isoflurano, havendo uma troca do átomo de cloro no carbono alfa da parte etil por um átomo de flúor. Então, há uma fluorinização que reduz a solubilidade, o volume de distribuição e a inflamabilidade e aumenta a estabilidade e a volatilidade. Desflurano: $\mathrm{CHF}_{2}-\mathrm{O}-\mathrm{CHF}-\mathrm{CF}_{3}$, Isoflurano $\mathrm{CHF}_{2}-\mathrm{O}-\mathrm{CHCl}$ $-\mathrm{CF}_{3}$

O desflurano tem peso molecular mais baixo e pressão de vapor a $20^{\circ}$ mais elevada do que os outros anestésicos haloge-

Revista Brasileira de Anestesiologia

Vol. 53, № 2, Março - Abril, 2003 nados em uso atualmente. Seu ponto de ebulição é muito bai$\mathrm{xo}\left(22,8^{\circ} \mathrm{C}\right)^{1}$. Isto significa que evapora facilmente à temperatura ambiente e que requer cuidados especiais de embalagem e de vaporização para não haver desperdício (Tabelal).

Tabela I - Algumas Propriedades Físicas do Desflurano em Relação a Outros Agentes Inalatórios ${ }^{1}$

\begin{tabular}{lccc}
\hline Anestésico & $\begin{array}{c}\text { Peso Molecular } \\
(\mathrm{g})\end{array}$ & $\begin{array}{c}\text { Pressão de Vapor } \\
\mathrm{mmHg} \mathrm{a} \mathrm{20}{ }^{\circ} \mathrm{C}\end{array}$ & $\begin{array}{c}\text { Ponto de Ebulição } \\
{ }^{\circ} \mathrm{C}\end{array}$ \\
\hline Desflurano & 168 & 600 & 22,8 \\
Sevoflurano & 200 & 170 & 58,5 \\
Óxido nitroso & 44 & - & - \\
Isoflurano & 184,5 & 240 & 48,5 \\
Enflurano & 184,5 & 172 & 56,2 \\
Halotano & 197,4 & 244 & 50,2 \\
\hline
\end{tabular}

Devido a estas propriedades, é mais estável, não sofre degradação metabólica em contato com cal sodada normalmente hidratada (15\%). Pode ocorrer formação de monóxido de carbono quando a cal sodada está seca ou pouco hidratada (abaixo de 4,8\%). A cal baritada seca produz mais monóxido de carbono do que a cal sodada também desidratada ${ }^{2}$.

As ligações flúor-carbono do desflurano são muito estáveis, como as do isoflurano, diferente do sevoflurano e enflurano que liberam grandes quantidades de flúor em forma de fluoretos orgânicos e inorgânicos ${ }^{2}$.

\section{Vaporização}

Algumas propriedades físicas do desflurano como o baixo ponto de ebulição $\left(22,8^{\circ} \mathrm{C}\right)$ e a alta pressão de vapor a $20^{\circ} \mathrm{C}$ $(669 \mathrm{mmHg}=88,5 \mathrm{Kpa})$ indicam que este agente é extremamente volátil e pode ser rapidamente transformado de líquido para vapor em temperatura ambiente acima do seu ponto de ebulição. Isto requer cuidados especiais quanto a sua embalagem e vaporização. Por esta razão, foram desenvolvidos vaporizadores diferentes dos que são utilizados para outros agentes.

O vaporizador "Tec 6" para desflurano tem dimensões de 11 $\mathrm{cm}$ de comprimento, $24,5 \mathrm{~cm}$ de altura e $25 \mathrm{~cm}$ de largura. Seu peso é de $9,5 \mathrm{~kg}$. As câmaras de líquido e de vapor são revestidas por forte blindagem, que são envolvidas por uma grade do sistema elétrico de aquecimento que permite passagens de ar em sua volta. Necessita eletricidade para manter as câmaras à temperatura de $39^{\circ} \mathrm{C}$. O líquido vai sendo vaporizado gradativamente e o vapor formado sai da sua câmara, fluindo até uma válvula de controle eletrônico para pressão do vapor e, a seguir, flui até uma outra válvula de pressão mecânica comandada pelo controle manual da concentração (dial). A partir deste ponto, segue para encontrar o fluxo diluente que passa pelo controle de um transdutor de pressão para evitar alterações de fluxo nas variações de diâmetros das vias de passagens de gás e vapor. Há um comando eletrônico entre o transdutor de pressão e a válvula que 
controla a saída do vapor. Isto permite a proporcionalidade entre fluxo de gás fresco e quantidade de vapor (Figura 1). O controle da concentração está graduado de $0 \%$ a 18\%, podendo variar de $1 \%$ entre $0 \%$ e $10 \%$ e $2 \%$ entre $10 \%$ e $18 \%$.

Para funcionar, após ligar o sistema elétrico, deve-se esperar acender luz que indica a obtenção da temperatura necessária para o aquecimento das câmaras.

Deve ser observado que este vaporizador não tem fluxo de borbulhamento. O vapor é obtido por aquecimento do líquido. Há um fluxo de gás que passa pelo vaporizador para receber o vapor formado; no entanto, nenhum fluxo penetra na câmara de onde o líquido é vaporizado, então só existe o fluxo diluente.

O abastecimento requer procedimento completamente vedado através da válvula de enchimento do vaporizador. $O$ vaporizador tem capacidade de $425 \mathrm{ml}$. Há visor com escala que indica o nível líquido de desflurano. Os intervalos entre os traços da escala representam $20 \mathrm{ml}$. O controle do nível líquido facilita a medida do consumo ${ }^{3}$.

\section{COEFICIENTES DE PARTIÇÃO - SOLUBILIDADE}

O desflurano tem baixa solubilidade que é expressa pelos seus coeficientes de partição nos meios orgânicos (Tabela II).

Sua baixa solubilidade lhe confere características farmacocinéticas muito desejáveis e também contribui com sua estabilidade (Tabela III).

Tabela II - Coeficientes de Partição Sangue/Gás e Cérebro/Gás dos Anestésicos Inalatórios ${ }^{2}$

\begin{tabular}{lcc}
\hline Anestésico & Coeficiente Sangue/Gás & Cérebro/Sangue \\
\hline Desflurano & 0,45 & 1,3 \\
Sevoflurano & 0,65 & 1,7 \\
Óxido Nitroso & 0,47 & 1,1 \\
Isoflurano & 1,4 & 1,6 \\
Enflurano & 1,8 & 1,3 \\
Halotano & 2,4 & 1,9 \\
\hline
\end{tabular}

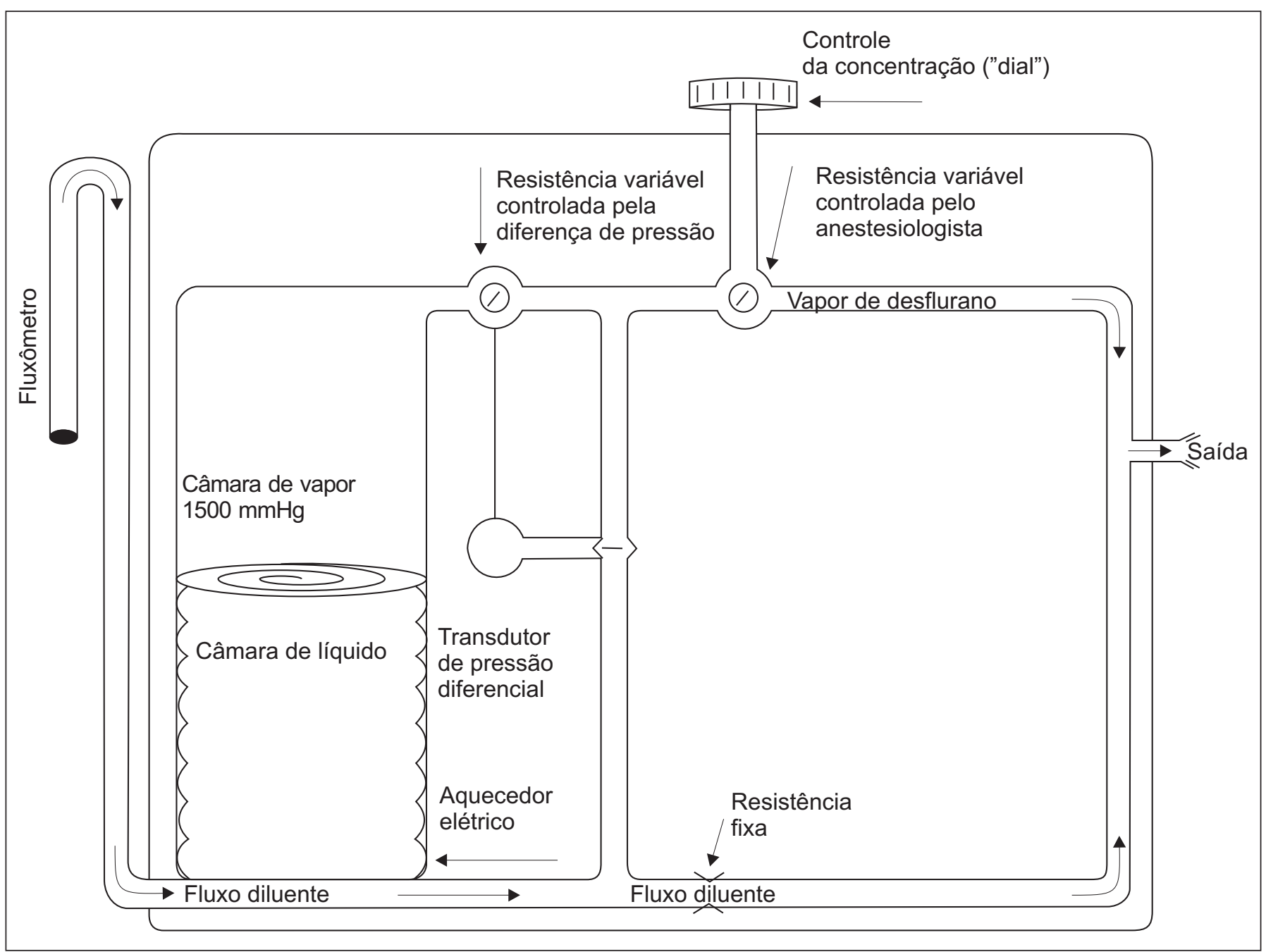

Figura 1 - Diagrama do TEC 6 
Tabela III - CAM, Percentagem de Degradação Metabólica da Quantidade Absorvida e Estabilidade nos Absorventes de Dióxido de Carbono (gás carbônico) dos Anestésicos Inalatórios ${ }^{1}$

\begin{tabular}{lccl}
\hline Anestésico & CAM & $\begin{array}{c}\text { Degradação } \\
\text { Metabólica } \\
(\%)\end{array}$ & $\begin{array}{l}\text { Estabilidade } \\
\text { Absorvente de } \\
\text { Gás Carbônico }\end{array}$ \\
\hline Desflurano & 6 & 0,02 & Estável \\
Sevoflurano & 2 & 3,0 & Instável \\
Oxido nitroso & 105 & 0,004 & Estável \\
Isoflurano & 1,15 & 0,2 & Estável \\
Enflurano & 2,0 & 2,4 & Estável \\
Halotano & 0,75 & 15 a 20 & Levemente instável \\
\hline
\end{tabular}

Sua potência é baixa, em pacientes adultos, de 30 a 60 anos e sua CAM, capaz de inibir a resposta de movimento a estímulo nociceptivos, é cerca de $6 \%$. Isto requer técnica de administração com fluxos mais baixos após saturar o sistema de inalação em alguns minutos ${ }^{4}$.

O desflurano é o agente inalatório que suporta as maiores temperaturas sem sofrer degradação metabólica. Em experiência realizada aquecendo a cal sodada em filtro de $580 \mathrm{ml}$ até a temperatura máxima de $80^{\circ} \mathrm{C}$, foi verificado que o desflurano não sofre degradação metabólica, enquanto o sevoflurano é degradado em $90 \%$ por hora e o halotano e o isoflurano em $20 \%$ e $18 \%$, respectivamente ${ }^{2,5}$.

\section{FARMACOCINÉTICA}

A captação, distribuição e eliminação do desflurano são dependentes da sua solubilidade no sangue e nos tecidos, e são determinantes da indução, manutenção e regressão da anestesia.

A progressão da concentração alveolar dos agentes anestésicos para entrar em equilíbrio com a concentração inspirada é sempre inversamente proporcional à solubilidade. No caso do desflurano, este processo ocorre muito rapidamente, devido a sua baixa solubilidade (Figura 2$)^{6}$.

Aindução da anestesia é muito rápida, comparada com a que é obtida com a administração de outros agentes mais solúveis.

Durante a manutenção da anestesia, tem se mostrado um agente muito estável e a diferença entre a concentração inspirada e a concentração administrada logo passa a ser muito pequena. O processo de equilíbrio vai se processando até que a concentração alveolar passa a ser muito próxima da concentração que sai do vaporizador.

A regressão da anestesia também é muito rápida, especialmente quando comparada a de outros agentes mais solúveis, notadamente os que têm coeficiente de partição cérebro/sangue mais elevado. Devido a esta característica farmacocinética, o desflurano é bem indicado sempre que se deseja um curto período de recuperação ou quando, por algum motivo, se deseja que o paciente possa emergir rapidamente da anestesia para que seja observada reação a algum estímulo, ou venha a obedecer a comando (Figura 3) ${ }^{6}$.

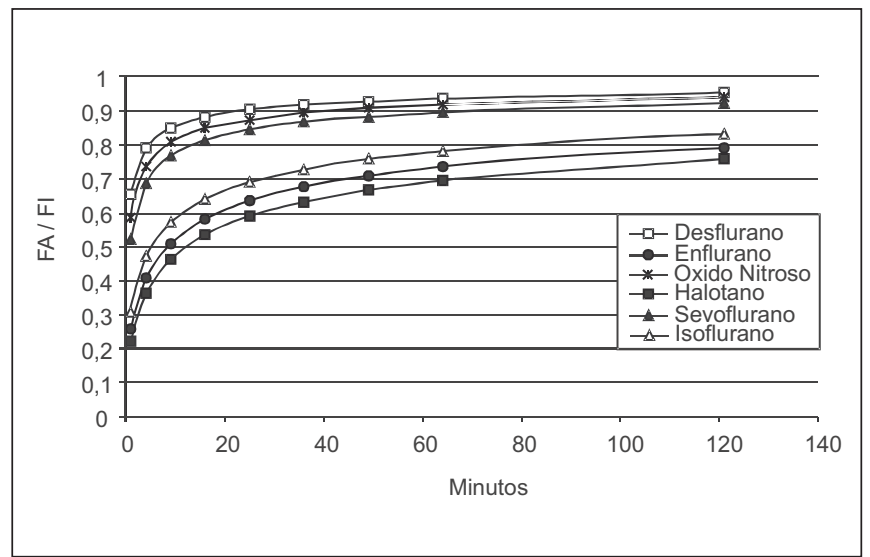

Figura 2 - Modelo Exponencial com Base na Captação

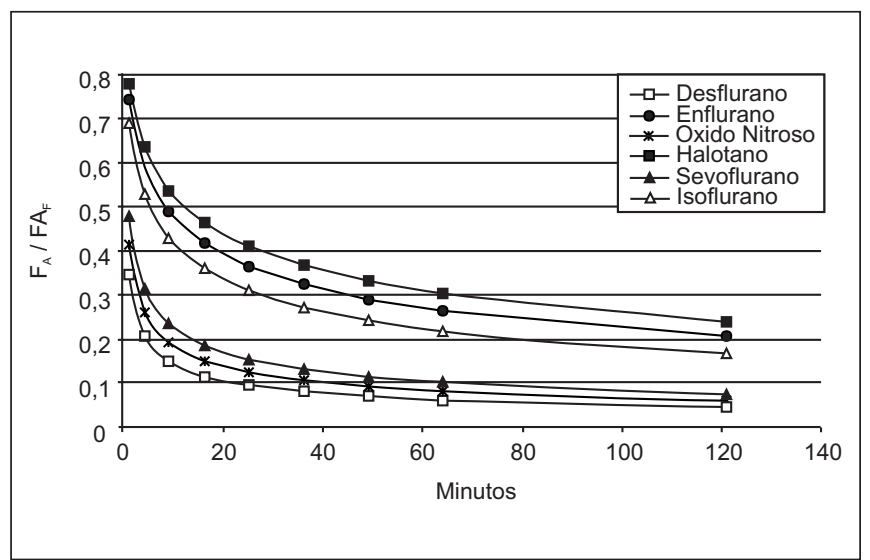

Figura 3 - Modelo Exponencial na Regressão da Anestesia

A eliminação rápida do desflurano favorece o despertar e a alta da sala de recuperação pós- anestésica em curto período e conseqüentemente o credencia como agente bem indicado em anestesia ambulatorial.

\section{FARMACODINÂMICA}

O desflurano é um agente que oferece boa estabilidade cardiovascular, não altera a função renal, não interfere no metabolismo hepático, é quase totalmente eliminado por via pulmonar e apenas $0,02 \%$ da quantidade absorvida é metabolizada. Por estas razões, é considerado um anestésico bem tolerado.

Do lado negativo, pode-se dizer que é irritante da via aérea pelo seu odor pungente, pode produzir maior incidência de vômito e aumenta o fluxo sangüíneo cerebral e pressão intracraniana em concentrações alveolares acima de $1 \mathrm{CAM}^{2}$.

\section{Ação sobre o Sistema Cardiovascular}

O desflurano aumenta a freqüência cardíaca, sendo este aumento dose-dependente, é mais acentuado a partir de 1 
CAM, diferente do isoflurano que aumenta muito até 1 CAM depois estabiliza. Mais diferente ainda do sevoflurano é o fato de que a freqüência diminui até 1 CAM depois aumenta. A pressão arterial média é reduzida também dose-dependente, semelhante ao isoflurano e diferente do sevoflurano que tende a estabilização após $1 \mathrm{CAM}^{7}$. O índice cardíaco é melhor mantido com o desflurano do que com os outros dois agentes, indicando que há sempre a compensação do débito cardíaco por aumento da freqüência cardíaca quando ocorre hipotensão arterial.

\section{Função Hepática e Função Renal}

O desflurano oferece os melhores resultados de avaliação clínico-laboratorial da função hepática (transaminases) e renal (depuração da creatinina) em relação a outros agentes anestésicos ${ }^{8}$, mostrando que pode ser utilizado mesmo na presença de doença hepática ou renal.

A liberação de flúor na forma iônica e de fluoretos é a menor entre os halogenados fluorados. Isto naturalmente favorece a melhor tolerância hepato-renal deste agente ${ }^{9,10}$.

\section{Ação sobre o Sistema Nervoso Central}

A ação principal do desflurano como agente anestésico, naturalmente é desenvolvida no sistema nervoso central. Sendo um agente considerado completo tem ação imobilizante preferentemente sobre a medula espinhal e ação amnésica e hipnótica sobre o cérebro. Estes efeitos são confirmados através da sua ação inibitória no potencial evocado somatossensitivo e índice bispectral do eletroencefalograma ${ }^{11}$. O desflurano não provoca convulsões e tem inclusive ação anticonvulsivante ${ }^{12}$. Sua ação sobre a resistência vascular cerebral, embora exista, é discutida em relação à dose. Há relatos de que esta só é significativa com conseqüente aumento da pressão intracraniana em concentrações alveolares acima de 1 CAM e quando existe grande massa tumoral, tendo na maioria das vezes um efeito semelhante ao do isoflurano ${ }^{13}$.

\section{Ação sobre o Aparelho Respiratório (Vias Aéreas)}

Outra ação adversa do desflurano que deve ser mencionada é a de irritante da via aérea devido ao odor pungente, produzindo tosse e conseqüente parada respiratória momentânea. Isto ocorre no início da indução anestésica sob máscara, podendo ser evitado pelo uso da indução venosa ou com um outro agente bem tolerado pela via aérea quando o paciente está consciente. Nas fases de manutenção e regressão da anestesia este efeito não ocorre ${ }^{2}$.

A exemplo dos outros anestésicos inalatórios, produz relaxamento muscular e depressão respiratória dose-dependente ${ }^{2}$

\section{OUTRAS AÇÕES}

Existem opiniões de que quando o anestésico inalatório é administrado em maiores concentrações favorece a deposição de grande volume nos espaços do tubo digestivo ocasionando náuseas e vômitos. Neste caso, o desflurano, a exemplo do óxido nitroso, tem esta maior probabilidade potencial; no entanto, a incidência de náuseas e vômitos é semelhante aos outros halogenados ${ }^{14}$.

\section{Metabolismo e Toxicidade}

Os estudos da toxicidade dos anestésicos inalatórios têm mostrado que esta se relaciona diretamente com o metabolismo destes agentes. Então, quanto mais são metabolizados mais reações tóxicas podem produzir. Nesse caso, é também muito importante a duração da exposição do organismo ao agente inalado, porque a quantidade absorvida certamente determina a porção desta que foi metabolizada. Deste modo, é importante saber a concentração do anestésico em unidades da CAM (múltiplo ou submúltiplo) e a duração em horas, assim se estabelece a expressão CAM/hora, que é o produto da concentração alveolar em CAM pelo número de horas de administração de anestésico.

Os halogenados fluorados que são metabolizados podem produzir o íon flúor $\left(\mathrm{F}^{-}\right)$que em si é tóxico, especialmente para o rim e fígado, e pode combinar com proteínas celulares gerando produtos potencialmente tóxicos ${ }^{15}$.

O desflurano tem o índice mais baixo de degradação metabólica entre os halogenados (Tabela III). Além disto, é considerado muito estável, ou seja, sua molécula não é facilmente alterada na presença de agentes físicos ou em contato com outras substâncias.

\section{Formação de Monóxido de Carbono}

Existe a possibilidade de formação de monóxido de carbono (CO) durante a administração da anestesia por halogenados, em sistema de inalação com absorção de gás carbônico, e conseqüente formação de carbohemoglobina ( $\mathrm{HbCO}$ ). Esta ocorrência tem sido descrita com o uso de todos os anestésicos inalatórios, porém o desflurano potencialmente seria o agente que formaria mais CO na presença da cal sodada. No entanto, isto só ocorre em níveis preocupantes quando a cal está desidratada (umidade $<4,6 \%$ ) e a temperatura se eleva a valores próximos a $70{ }^{\circ} \mathrm{C}{ }^{16}$. Estas condições dificilmente são encontradas durante o procedimento clínico da anestesia, em que a cal está hidratada (contém cerca de $15 \%$ de água), e a temperatura no sistema de inalação não excede muito a temperatura corporal $\left(37^{\circ} \mathrm{C}\right)$.

O monóxido de carbono (CO) tem cerca de 200 vezes mais afinidade pela hemoglobina $(\mathrm{Hb})$ do que o oxigênio. A sua combinação com a hemoglobina forma a $\mathrm{HbCO}$, que é um pigmento cor-de-rosa e passa despercebido; no entanto, produz os efeitos da anemia, ou seja, desloca a curva da hemoglobina para esquerda e tem repercussões clínicas como taquicardia e grande aumento do tempo de coagulação do sangue ${ }^{17}$.

Em caso de suspeita na formação de monóxido de carbono, com grande aumento na temperatura do reservatório de cal sodada, deve ser administrado oxigênio a $100 \%$ e aumenta- 
do o fluxo de gás fresco para 2 vezes o volume minuto respiratório a fim de lavar completamente o sistema de inalação e deslocar o $\mathrm{CO}$ da $\mathrm{Hb}$ e em seguida ser eliminado ${ }^{17-19}$.

Há relatos experimentais de que a formação de CO pelo contato dos agente anestésicos inalatórios e cal sodada, até uma concentração inspirada (FI) de $1 \%$ ou um pouco acima disso, já apresenta risco de intoxicação. No entanto, após 10 minutos, esta $\mathrm{FI}$ é reduzida espontaneamente e gradativamente a níveis próximos de zero ${ }^{16}$. Esse fenômeno se relaciona à saturação dos componentes de hidróxido de sódio e hidróxido de potássio da cal ${ }^{22}$.

Deve ser esclarecido que estes trabalhos experimentais são realizados sempre com a cal sodada desidratada ${ }^{20,21}$ e que há relatos de casos clínicos com vários anestésicos; entretanto, na maioria das vezes com uso da cal baritada supostamente desidratada.

Há comprovação experimental de que pequena alteração na composição química da cal sodada, eliminando as bases fortes, os hidróxidos de sódio e de potássio, reduz significantemente a formação de monóxido de carbono sem prejuízo para absorção do dióxido de carbono ${ }^{22}$.

\section{APLICAÇÃO CLÍNICA}

\section{Técnicas de Administração}

As propriedades físico-químicas e as características farmacocinéticas e farmacodinâmicas são determinantes do uso clínico do desflurano. Deste modo, tendo ponto de ebulição baixo volatiliza facilmente as temperaturas usuais nas salas de operação e sua CAM elevada requer que seja administrado em altas concentrações; então, é recomendável o uso de fluxo de gás fresco baixo para que a sua aplicabilidade clínica seja economicamente viável. Além disto, o uso de um agente coadjuvante, como o óxido nitroso reduz a sua CAM e possibilita que seja administrado em menores concentrações.

Está muito difundida uma técnica que utiliza um fluxo de gás fresco de 5 L. $\mathrm{min}^{-1}$, oxigênio (2 L. $\mathrm{min}^{-1}$ ) e óxido nitroso (3 L. min $^{-1}$ ) durante 5 minutos e com concentração administrada no vaporizador a 1,5 CAM. Após este período, o fluxo é reduzido para $1 \mathrm{~L} \cdot \mathrm{min}^{-1}\left(0,6 \mathrm{~L} \cdot \mathrm{min}^{-1}\right.$ de óxido nitroso $0,4 \mathrm{~L}$. $\mathrm{min}^{-1} \mathrm{de}$ oxigênio) e a concentração administrada para $1 \mathrm{CAM}^{1}$.

Recentemente foi descrita uma técnica de "seqüência de fluxos de gases frescos para anestesia com baixo fluxo", que utiliza o fluxo de gás fresco de oxigênio de $3,5 \mathrm{~L}$. $\mathrm{min}^{-1}$ durante 1 minuto e depois $1 \mathrm{~L} \cdot \mathrm{min}^{-1}$. A concentração administrada de 3 CAM (18\%) em 1 minuto, depois 1,5 CAM em 10 minutos e 1,2 CAM a seguir. A concentração alveolar obtida é sempre muito próxima de $1 \mathrm{CAM}^{4}$.

\section{Principais Indicações}

O desflurano é um agente de grande aplicabilidade em procedimentos anestésicos ambulatoriais devido aos curtíssimos tempos de indução e emergência (2 a 3 minutos para ambos) ${ }^{6}$. Sua baixa solubilidade e mínima metabolização permitem que seja administrado por longo período com bai- xíssima toxicidade e grande tolerabilidade do organismo. Sendo, por estas razões, bem indicado em pacientes idosos e também em pacientes com hepatopatias e nefropatias. Pode ser usado com grande margem de segurança em anestesia para cirurgia cardiovascular, considerando-se que oferece boa estabilidade hemodinâmica e não é arritmogênico.

Em anestesia de longa duração, é sempre muito considerado o potencial de toxicidade do agente anestésico utilizado, que fica por período prolongado no organismo do paciente. Deste modo, sendo o desflurano o anestésico inalatório menos metabolizado e tendo baixa solubilidade, oferece esta vantagem, podendo ser bem indicado para procedimentos demorados ${ }^{23}$.

O desflurano pode ser bem indicado em pacientes com cardiopatias. Tem ação taquicardizante quando usado em concentrações alveolares acima de 1 CAM; no entanto, a adição de óxido nitroso permite que a anestesia seja mais profunda sem alterar a estabilidade cardiovascular. Da mesma forma, o uso prévio de fentanil inibe esta tendência a aumento da freqüência cardíaca ${ }^{24}$.

Há situações em que se deseja que o paciente possa emergir rapidamente da anestesia, na maioria das vezes para avaliação de parâmetros fisiológicos, como, por exemplo, o clássico Wake up test em cirurgia da coluna vertebral. Além disto, em anestesias para cirurgias ambulatoriais, deseja-se que o paciente recupere a consciência o mais breve possível. Nessas situações, o desflurano é um agente muito bem indicado ${ }^{24}$. Paciente idosos toleram melhor agentes menos solúveis porque permanecem menos em seus organismos e deste modo interferem menos em suas atividades metabólicas celulares, especialmente em órgãos nobres como os componentes do sistema nervoso central ${ }^{25}$. Por esta razão, o desflurano é bem indicado para uso em anestesias destes pacientes que devem recuperar suas funções mentais o mais cedo possível ${ }^{26}$.

\section{Precauções e Possíveis Contra-Indicações}

Em neurocirurgia intracraniana, deve ser usado com cautela especialmente quando existem grandes massas tumorais e aumento da pressão intracraniana. É recomendável não atingir concentração alveolar acima de 1 CAM e não usar óxido nitroso como agente coadjuvante ${ }^{13}$.

Em anestesia pediátrica, pode ser usado com a vantagem de permitir aprofundar e superficializar a anestesia. No entanto, deve ser administrado com um agente de indução venoso ou outro agente inalatório de odor melhor tolerado como o halotano ou sevoflurano ${ }^{27}$.

O uso do baixo fluxo oferece a vantagem de menor consumo, menor poluição e melhor umidificação do sistema de inalação e da via aérea; no entanto, deve-se ter o cuidado permanente de evitar misturas hipóxicas. Desde modo, quando se faz opção pelo fluxo basal que é administração de um fluxo de oxigênio semelhante ao consumo e é acrescido um fluxo adicional em igual valor, ou pouco maior, de óxido nitroso, é importante saber que o acúmulo de nitrogênio que ocorre 
normalmente com o uso do baixo fluxo diluirá ainda mais o oxigênio no alvéolo pulmonar ${ }^{28}$. É mandatória a monitorização da $\mathrm{F}_{1} \mathrm{O}_{2}$ e $\mathrm{FEO}_{2}$.

Além disto, caso exista suspeita da cal sodada estar desidratada, deve-se colocar água na parte superior do reservatório (5 $\mathrm{ml}$ para cada $100 \mathrm{ml}$ de cal), assim evitará a formação de monóxido de carbono ${ }^{20,21}$

\section{CONSUMO E CUSTOS}

O consumo de um agente é o principal determinante do seu custo. O desflurano é muito volátil e tem CAM elevada; então, o seu consumo deve ser reduzido para que o custo seja acessível ao mercado consumidor. Um dos elementos mais importantes na composição do consumo de um anestésico inalatório é o fluxo de gás fresco. Então as técnicas que utilizam o baixo fluxo são muito importantes para administrar este agente.

\section{Observando-se a fórmula que calcula o consumo:}

$$
\text { Consumo }=\mathrm{C} \times \mathrm{F} \times \mathrm{T} / \mathrm{D} \times \mathrm{Vm} \times \mathrm{TA}+\mathrm{t}^{\circ} \mathrm{C} / \mathrm{PM} \times 273
$$

Onde: $\mathrm{C}=$ Concentração em volumes \%

$\mathrm{F}=$ Fluxo em $\mathrm{ml} \cdot \mathrm{min}^{-1}$

$\mathrm{T}=$ Tempo em minutos

$\mathrm{D}=$ Densidade

$\mathrm{VM}=$ Volume molecular em litros $(22,4 \mathrm{I})$

TA $=$ Temperatura absoluta $\left(273^{\circ}\right)$

$\mathrm{T}^{\circ} \mathrm{C}=$ Temperatura ambiente em graus centígrados

$\mathrm{PM}=$ Peso molecular

Verifica-se que o consumo é diretamente proporcional ao fluxo, à concentração e ao tempo e inversamente proporcional à relação densidade/peso molecular $(\mathrm{PM})^{4}$.

A tabela IV mostra que usando fluxos baixos (1 L. $\left.\mathrm{min}^{-1}\right)$ na manutenção da anestesia, após saturação do sistema inalatório do aparelho de anestesia, é possível obter um consumo pequeno para resultar em um custo acessível ${ }^{4}$. Estes dados são confirmados por outros autores ${ }^{29,30}$.

Tabela IV - Consumo de Anestésico Inalatório com Baixo Fluxo de Gases ${ }^{4}$.

\begin{tabular}{lc}
\hline Anestésico & $\begin{array}{c}\text { Média do Volume Gasto }(\mathrm{ml}) \\
\text { nos 20 }\end{array}$ \\
\hline Halotanutos Iniciais
\end{tabular}

\section{CONCLUSÃO}

O desflurano representa mais uma evolução em direção ao anestésico ideal. Suas propriedades físico-químicas conferem-Ihe características farmacocinéticas bastante desejá- veis, como a indução (progressão) e regressão muito rápidas e também a metabolização mínima com baixíssima toxicidade orgânica e forte estabilidade molecular, inclusive na presença de absorventes de dióxido de carbono.

Suas ações farmacodinâmicas são perfeitamente toleradas e compatíveis com o seu uso clínico.

Tendo-se as precauções quanto à vaporização, armazenamento e consumo, o desflurano pode ser usado inclusive em larga escala especialmente em anestesia ambulatorial, pacientes graves, hepatopatas, nefropatas, idosos e em procedimentos de longa duração.

\section{Desflurane: Physicochemical Properties, Pharmacology and Clinical Use}

Renato Ângelo Saraiva, TSA, M.D.

\section{INTRODUCTION}

After the development of nuclear chemistry in the 40's and 50 's of the $20^{\text {th }}$ Century, there has been a major evolution in the synthesis of inhalation anesthetics as from hydrocarbons. Halogenation of those compounds, which consists in replacing hydrogen atoms by fluorine or chlorine atoms, results in the formation of a substance with anesthetic properties. Several of those agents were clinically tested and some had wide practical applications.

Currently, halothane, enflurane, isoflurane, sevoflurane and desflurane are being clinically used. The two latter were more recently introduced.

There is still no ideal agent, fulfilling all desired properties without undesirable effects. For this reason, there are always new agents being investigated. From halogenates currently being used, halothane is still very popular, especially in pediatrics, although being able to induce arrhythmia's and develop potential toxicity due to its instability worsened by its high solubility which favors more absorption and, as a consequence, major metabolization. Isoflurane is not well tolerated for inhalation induction and does not have a very fast anesthetic recovery. Even though, if well tolerated by the organism, it is probably today the most widely used inhalational anesthetic agent. Sevoflurane has a pleasant odor and does not induce arrhythmia's. For this reason it is being widely used in pediatric anesthesia, especially in inhalation indications. However, it releases too much fluorine and, for this reason, it is not recommended for long procedures, especially in renal failure patients.

Desflurane has a poor blood and tissue solubility thus being promptly absorbed and excreted. It favors alveolar concentration progression, which is soon close to MAC and, at administration completion, allows alveolar concentration to be sub-anesthetic almost immediately, allowing for a very fast anesthetic emergence. It is minimally metabolized and serum fluorine release is negligible. This gives it the status of Vol. 53, N 2, Março - Abril, 2003 
the best tolerated agents by the body in terms of toxicity. Its pungent odor severely limits its application in inhalation induction. So, it is to be imagined that desflurane is gaining space as fast progression and regression agent with very low toxicity, but loses ground in inhalational induction and in the requirement of strict soda lime humidity to prevent the formation of carbon monoxide.

Clearly desflurane, although not being an agent very close to what could be called ideal, represents some steps in this direction.

This study aimed at describing physicochemical and pharmacological characteristics of desflurane in addition to reporting results of its clinical application.

\section{PHYSICOCHEMICAL PROPERTIES}

Desflurane molecular structure is similar to isoflurane with replacement of the chlorine atom, in the alpha carbon of the ethyl part, by a fluorine atom. There is then fluorinization which decreases solubility, distribution volume and flammability while increases stability and volatility.

Desflurane: $\mathrm{CHF}_{2}-\mathrm{O}-\mathrm{CHF}-\mathrm{CF}_{3}$, Isoflurane: $\mathrm{CHF}_{2}-\mathrm{O}-\mathrm{CH}$ $\mathrm{Cl}-\mathrm{CF}_{3}$

Desflurane has lower molecular weight and higher vapor pressure at $20^{\circ}$ than other halogenates currently being used. Its boiling point is very low $\left(22.8^{\circ} \mathrm{C}\right)^{1}$ meaning that it evaporates very easily at room temperature and requires special care with packaging and vaporization to avoid waste (Table I).

Table I - Some Desflurane Physical Properties as Compared to Other Inhalation Agents ${ }^{1}$

\begin{tabular}{lccc}
\hline Anesthetic Agent & $\begin{array}{c}\text { Molecular Weight } \\
(\mathrm{g})\end{array}$ & $\begin{array}{c}\text { Vapor Pressure } \\
\mathrm{mmHg} \text { at } 20^{\circ} \mathrm{C}\end{array}$ & $\begin{array}{c}\text { Boiling Point } \\
{ }^{\circ} \mathrm{C}\end{array}$ \\
\hline Desflurane & 168 & 600 & 22.8 \\
Sevoflurane & 200 & 170 & 58.5 \\
Nitrous oxide & 44 & - & - \\
Isoflurane & 184.5 & 240 & 48.5 \\
Enflurane & 184.5 & 172 & 56.2 \\
Halothane & 197.4 & 244 & 50.2 \\
\hline
\end{tabular}

Due to these properties, it is more stable and does not undergo metabolic degradation in contact with normally hydrated soda lime (15\%). There might be carbon monoxide formation in the presence of dry or poorly hydrated (below $4.8 \%$ ) soda lime. Dry barite lime produces more carbon monoxide as compared to also dehydrated soda lime ${ }^{2}$.

Desflurane fluorine-carbon bindings are very stable, as with isoflurane and differently from sevoflurane and enflurane, which release high amounts of fluorine in the form of organic and inorganic fluoride ${ }^{2}$.

\section{VAPORIZATION}

Some desflurane physical properties, such as low boiling point $\left(22.8^{\circ} \mathrm{C}\right)$ and high vapor pressure at $20^{\circ} \mathrm{C}(669 \mathrm{mmHg}=$

Revista Brasileira de Anestesiologia

Vol. 53, № 2, Março - Abril, 2003
$88.5 \mathrm{Kpa}$ ) indicate that this agent is extremely volatile and may be promptly transformed from liquid to vapor in room temperatures above its boiling point. This requires special care with packaging and vaporization. For this reason, vaporizers were developed which are different from those used for other agents.

Desflurane "Tec 6" vaporizer is $11 \mathrm{~cm}$ long, $24.5 \mathrm{~cm}$ high and $25 \mathrm{~cm}$ wide, weighing $9.5 \mathrm{~kg}$. Vapor and liquid chambers are coated with strong shielding and are involved by an electric heating system grid which allows the air to flow around it. Energy is needed to maintain chambers at a temperature of $39^{\circ} \mathrm{C}$. The fluid is gradually vaporized and the vapor formed exits its chamber flowing to the electronic vapor pressure control valve and then to another mechanical pressure valve commanded by the manual concentration control (dial). From this point, it proceeds to meet the diluent flow which passes through a pressure transducer to prevent flow changes in different diameters of gas and vapor tubes. There is an electronic command between the pressure transducer and the valve controlling vapor exit. This allows a balance between fresh gas flow and vapor (Figure 1).

Concentration control is graded $0 \%$ to $18 \%$ and may vary $1 \%$ between $0 \%$ and $10 \%$, and $2 \%$ between $10 \%$ and $18 \%$.

To operate, after turning the machine on, one should wait for the light indicating that the temperature needed to heat the chambers was achieved.

This vaporizer has no bubbling flow. Vapor is obtained by heating the fluid. There is gas flow passing through the vaporizer to receive the produced vapor; however, no flow enters the chamber from which the fluid is vaporized, so there is only diluent flow.

Replenishment requires a sealed procedure through the vaporizer's filling valve.

Vaporizer's capacity is $425 \mathrm{ml}$. There is check-window with a scale indicating desflurane liquid level. The scale is graded in

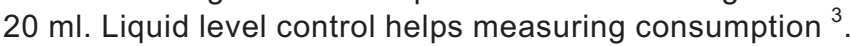

\section{PARTITION - SOLUBILITY COEFFICIENT}

Desflurane has low solubility which is expressed by its partition coefficients in organic media (Table II).

Table II - Blood/Gas and Brain/Gas Partition Coefficients of Inhalation Anesthetics ${ }^{2}$

\begin{tabular}{lcc}
\hline Anesthetic Agent & Blood/Gas Coefficient & Brain/Gas \\
\hline Desflurane & 0.45 & 1.3 \\
Sevoflurane & 0.65 & 1.7 \\
Nitrous oxide & 0.47 & 1.1 \\
Isoflurane & 1.4 & 1.6 \\
Enflurane & 1.8 & 1.3 \\
Halothane & 2.4 & 1.9 \\
\hline
\end{tabular}

Its low solubility gives it well desirable pharmacokinetic characteristics and also contributes to its stability (Table III). 
Table III - MAC, Percentage of Metabolic Degradation of Absorbed Amount and Stability of Inhalation Anesthetics Carbon Dioxide Absorbents ${ }^{1}$

\begin{tabular}{lccl}
\hline $\begin{array}{l}\text { Anesthetic } \\
\text { Agent }\end{array}$ & $\begin{array}{c}\text { MAC } \\
(\%)\end{array}$ & $\begin{array}{c}\text { Metabolic } \\
\text { Degradation } \\
(\%)\end{array}$ & $\begin{array}{l}\text { Carbon Dioxide } \\
\text { Absorbent Stability }\end{array}$ \\
\hline Desflurane & 6 & 0.02 & Stable \\
Sevoflurane & 2 & 3.0 & Unstable \\
Nitrous oxide & 105 & 0.004 & Stable \\
Isoflurane & 1.15 & 0.2 & Stable \\
Enflurane & 2.0 & 2.4 & Stable \\
Halothane & 0.75 & 15 to 20 & Slightly unstable \\
\hline
\end{tabular}

It has low potency in adult patients aged 30 to 60 years and its MAC, able to inhibit movement response to noxious stimulations, is approximately $6 \%$. This requires lower flow administration after saturating the inhalation system for some minutes ${ }^{4}$. Desflurane is the inhalational agent resisting the highest temperatures without undergoing metabolic degradation. In an experiment with soda lime heated in a $580 \mathrm{ml}$ fil- ter to the maximum temperature of $80^{\circ} \mathrm{C}$, it was observed that desflurane has not undergone metabolic degradation, while sevoflurane was degraded $90 \%$ per hour and halothane and isoflurane were degraded $20 \%$ and $18 \%$, respectively ${ }^{2,5}$.

\section{PHARMACOKINETICS}

Desflurane uptake, distribution and excretion depend on its blood and tissue solubility and are factors determining anesthetic induction, maintenance and recovery.

Anesthetic agents alveolar concentration progression to meet inspired concentration is always inversely proportional to solubility. This process is very fast with desflurane due to its low solubility (Figure 2) ${ }^{6}$.

Anesthetic induction is very fast as compared to other more soluble agents.

During anesthetic maintenance desflurane has proven to be a very stable agent and the difference between inspired and administered concentration soon becomes very small. There is a constant equilibrium process until alveolar concentration becomes very close to the concentration leaving the vaporizer.

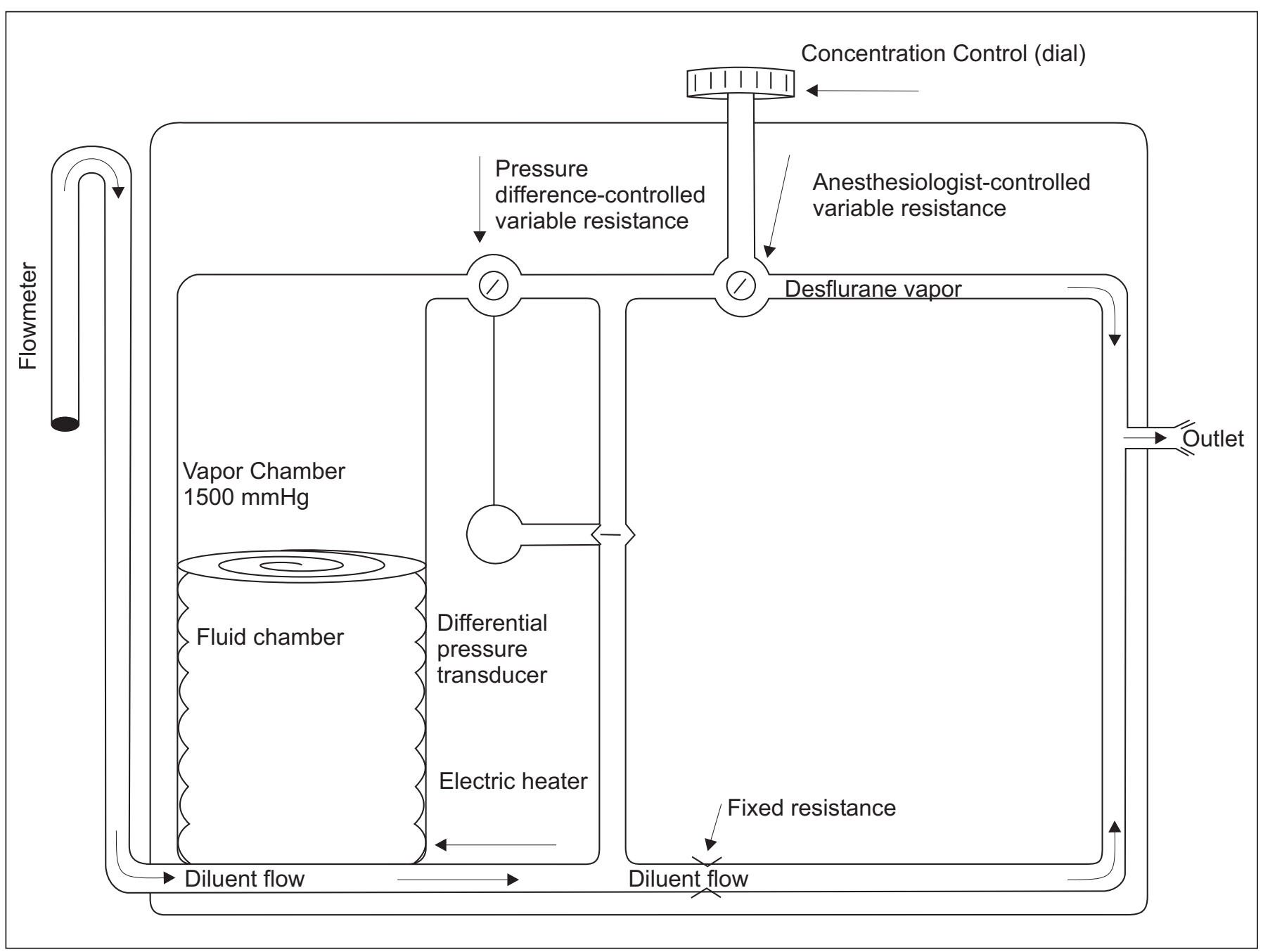

Figure 1 -TEC 6 Diagram 


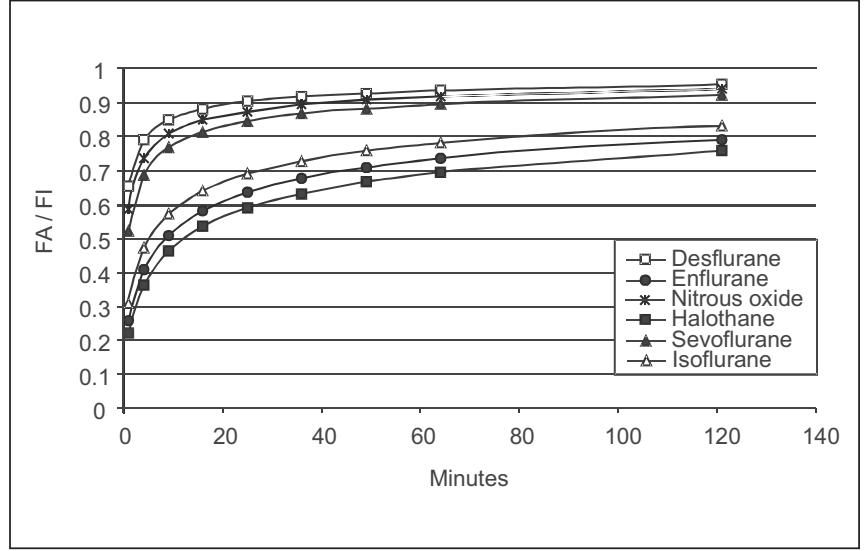

Figure 2 - Exponential Model Based on Uptake

Anesthetic recovery is also very fast, especially as compared to other more soluble agents, especially those with higher brain/blood partition coefficient. Due to this pharmacokinetic characteristic, desflurane is a good indication whenever a fast recovery is desired or when, for some reason, it is desirable that patients promptly emerge from anesthesia for the observation of some stimulation, or to respond to a command (Figure 3$)^{6}$.

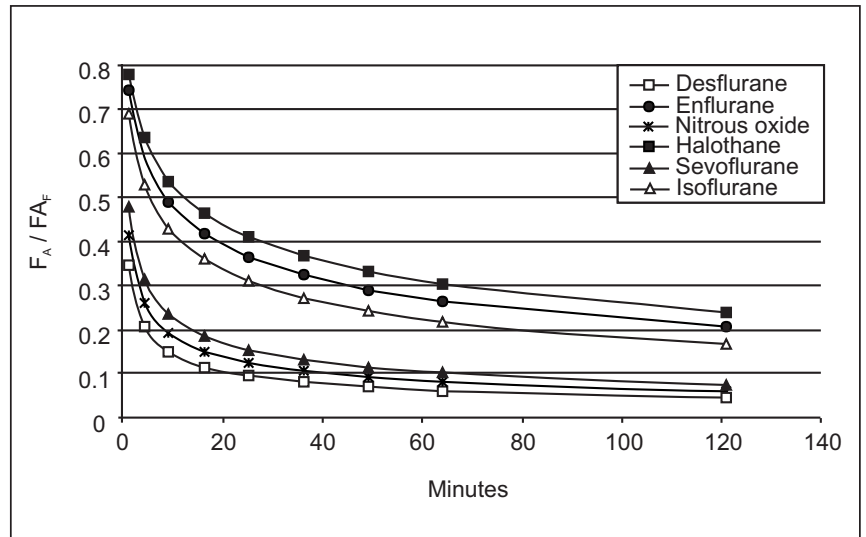

Figure 3 - Exponential Model in Anesthetic Recovery

Fast desflurane excretion favors emergence and PACU faster discharge and, as a consequence, it is a good choice for outpatient procedures.

\section{PHARMACODYNAMICS}

Desflurane provides good cardiovascular stability, does not change renal function, does not interfere with liver metabolism and is almost totally excreted by the lungs; only $0.02 \%$ of the absorbed amount is metabolized. For those reasons, it is considered a well tolerated anesthetic agent.

On the negative side, one may say that it irritates airways with its pungent odor, may increase the incidence of vomiting and increase brain blood flow and intracranial pressure in alveolar concentrations above $1 \mathrm{MAC}^{2}$.

\section{Cardiovascular Effects}

Desflurane increases heart rate in a dose-dependent manner being higher as from $1 \mathrm{MAC}$, which is different from isoflurane which steeply increases until 1 MAC and then stabilizes. Even more different than sevoflurane is the fact that the rate decreases until $1 \mathrm{MAC}$ and then increases. Mean blood pressure is decreased also in a dose-dependent manner, similar to isoflurane and different from sevoflurane which tends to stabilize as from $1 \mathrm{MAC}^{7}$. Cardiac index is better maintained with desflurane as compared to the other two agents, indicating that there is always cardiac output compensation by increased heart rate in the presence of arterial hypotension.

\section{Liver Function and Renal Function}

Desflurane offers the best clinical-lab evaluation results of liver function (transaminases) and renal function (creatinine clearance) as compared to other anesthetic agents ${ }^{8}$, showing that it may be used even in the presence of liver or renal disease.

Ion fluorine and fluoride release is the lowest among fluorinated halogenates. This naturally favors better liver-renal tolerance of this agent ${ }^{9,10}$.

\section{Central Nervous System Effects}

Major desflurane action is developed in the central nervous system. Being considered a complete agent, it acts by immobilizing preferably spinal cord and has amnesic and hypnotic effect on the brain. These effects are confirmed through the inhibitory action on somatosensory evoked potential and EEG bispectral index ${ }^{11}$.

Desflurane does not induce seizures being even anticonvulsivant ${ }^{12}$. Although present, its action on brain vascular resistance is discussed regarding the dose. There are reports that it would only be significant, with a consequent increase in intracranial pressure in alveolar concentrations above $1 \mathrm{MAC}$ and when there is a large tumor, having, most of the times, an effect similar to isoflurane ${ }^{13}$.

\section{Respiratory System Effects (Airways)}

Another desflurane adverse effect to be mentioned is airways irritation by its pungent odor, producing cough and consequent transient respiratory arrest. This is seen in the beginning of anesthetic induction under mask and may be prevented with intravenous induction or the use of an agent well tolerated by airways when the patient is awaken. There is no such effect during anesthetic maintenance and recovery ${ }^{2}$. Similar to other inhalational anesthetics, desflurane produces a dose-dependent muscle relaxation and respiratory depression ${ }^{2}$. 


\section{OTHER EFFECTS}

Some authors believe that when an inhalational anesthetic drug is administered in higher concentrations, it favors the deposition of large volumes in digestive tube spaces leading to nausea and vomiting. In this case desflurane, similar to nitrous oxide, would have this potential; however, the incidence of nausea and vomiting is similar to other halogenate agents ${ }^{14}$.

\section{Metabolism and Toxicity}

Inhalation anesthetics toxicity studies have shown that it is directly related to the metabolism of such agents. So, the more they are metabolized, more toxic reactions they may produce. In this case it is also important the duration of the exposure to the inhaled agent, since the absorbed amount certainly determines the amount to be metabolized. So, it is important to know anesthetic concentrations in MAC units (multiple or submultiple) and the duration in hours. This way it is possible to obtain the expression MAC/hour which is the product of alveolar concentration in MAC multiplied by the number of anesthetic administration hours.

Metabolized fluorinated halogenates may produce fluorine (F) which is toxic in itself, especially for kidneys and liver, and may combine with cell proteins to generate potentially toxic products $^{15}$

Desflurane has the lowest metabolic degradation ratio among halogenates (Table III). In addition, it is considered very stable, that is, its molecule is not easily changed in the presence of physical agents or in contact with other substances.

\section{Carbon Monoxide Formation}

There is the possibility for carbon monoxide formation (CO) during halogenate anesthetic induction in an inhalation system with carbon dioxide absorption and consequent formation of carboxyhemoglobin ( $\mathrm{HbCO}$ ). This has been described with all inhalation anesthetics, however desflurane would potentially be the agent forming more $\mathrm{CO}$ in the presence of soda lime. However, this only occurs in dangerous levels when soda lime is dehydrated (humidity $<4.6 \%$ ) and temperature is increased to levels close to $70^{\circ} \mathrm{C}{ }^{16}$. These conditions are seldom found during anesthesia in which soda lime is hydrated (with approximately $15 \%$ of water) and inhalational system temperature does not exceed body temperature (37 $\left.{ }^{\circ} \mathrm{C}\right)$ too much.

Carbon monoxide (CO) has approximately 200 times more affinity with hemoglobin $(\mathrm{Hb})$ than oxygen. When combined to hemoglobin it forms $\mathrm{HbCO}$ which is an unnoticed pink pigment; however it has the effects of anemia, that is, shifts hemoglobin curve to the left and has clinical repercussions such as tachycardia and a major increase in blood coagulation time ${ }^{17}$.

If there is a suspicion of carbon monoxide formation with major increase in soda lime reservoir's temperature, 100\% oxy- gen should be administered and fresh gas flow should be increased to twice respiratory minute volume to totally washout the inhalation system and separate $\mathrm{CO}$ from $\mathrm{Hb}$ to be then excreted $^{17-19}$.

There are experimental reports stating that $\mathrm{CO}$ formation by contact with inhalation anesthetics and soda lime, up to an inspired concentration (FI) of $1 \%$ or a little above, already poses intoxication risks. However, after 10 minutes, this FI is spontaneously and gradually decreased to levels close to zero ${ }^{16}$. This phenomenon is related to lime's sodium hydroxide and potassium hydroxide components saturation ${ }^{22}$. it has to be explained that such experimental trials are always carried out with dehydrated soda lime ${ }^{20,21}$ and that there are reports on clinical cases with different anesthetic agents, however most of the times with supposedly dehydrated barite lime.

There are experimental evidences that minor changes in soda lime chemical composition by eliminating strong bases, sodium and potassium hydroxide, would significantly decrease the formation of carbon monoxide without impairing carbon dioxide absorption ${ }^{22}$.

\section{CLINICAL APPLICATION}

\section{Administration Techniques}

Physicochemical properties and pharmacokinetic and pharmacodynamic characteristics determine desflurane clinical use. For having a low boiling point, it is easily volatized at usual operating room temperatures and its high MAC requires it to be administered in high concentrations; so, it is desirable the use of low fresh gas flows for it to be economically feasible. In addition, a coadjuvant agent, such as nitrous oxide, decreases MAC and allows its administration in lower concentrations.

A widespread technique uses 5 L. $\mathrm{min}^{-1}$ fresh gas flow, oxygen $\left(2 \mathrm{~L} \cdot \mathrm{min}^{-1}\right)$ and nitrous oxide $\left(3 \mathrm{~L} \cdot \mathrm{min}^{-1}\right)$ for 5 minutes with 1.5 MAC vaporizer concentration. After this period, the flow is decreased to $1 \mathrm{~L}$. $\mathrm{min}^{-1}$ (0.6 L. $\mathrm{min}^{-1}$ nitrous oxide, $0.4 \mathrm{~L}$. $\mathrm{min}^{-1}$ oxygen) and administered concentration is decreased to 1 MAC ${ }^{1}$.

Recently, a technique with "fresh gas flow sequences for low flow anesthesia" has been described, using oxygen fresh gas flow of $3.5 \mathrm{~L}$. $\mathrm{min}^{-1}$ for 1 minute and $1 \mathrm{~L}$. $\mathrm{min}^{-1}$ thereafter. Administered concentration of $3 \mathrm{MAC}(18 \%)$ in 1 minute, then 1.5 MAC in 10 minutes and 1.2 MAC thereafter. Alveolar concentration is always very close to $1 \mathrm{MAC}^{4}$.

\section{Major Indications}

Desflurane is widely applicable in outpatient anesthetic procedures due to its very fast induction and emergence times (2 to 3 minutes for both) ${ }^{6}$. Its low solubility and minimum metabolization allow it to be administered for a long period with very low toxicity and good body tolerance. For these reasons, it is a good indication for elderly patients or liver or renal Vol. 53, № 2, Março - Abril, 2003 
disease patients. It may be used with a wide safety margin in anesthesia for cardiovascular surgeries, because it provides good hemodynamic stability and does not induce arrhythmia's.

For long procedures, toxicity potential of anesthetic agents remaining for a long period in patients' body is always considered. Desflurane, for being the less metabolized inhalational anesthetic agent with low solubility has this advantage and may be indicated for long procedures ${ }^{23}$.

Desflurane is a good indication for cardiac patients. It induces tachycardia when used in concentrations above 1 MAC; however, the association of nitrous oxide allows for a deeper anesthesia without cardiovascular changes. Similarly, this trend to increased heart rate is inhibited by fentanyl. There are situations in which a fast emergence is desirable, mostly for physiological parameters evaluation, such as the classic Wake up test in spinal surgeries. In addition, a fast consciousness recovery is desirable in outpatient procedures. For such situations, desflurane is a very good choice $^{24}$.

Elderly patients tolerate better less soluble agents because they remain in their bodies for a shorter period thus interfering less with their cell metabolic functions, especially in noble organs such as central nervous system components ${ }^{25}$. So, desflurane is a good indication for such patients who need to recover their mental functions as soon as possible ${ }^{26}$.

\section{Precautions and Potential Counterindications}

It should be carefully used in intracranial surgeries, especially in the presence of large tumors and increased intracranial pressure. The recommendation is to avoid alveolar concentrations above $1 \mathrm{MAC}$ and nitrous oxide as coadjuvant agent ${ }^{13}$.

It may be used in pediatric anesthesia with the advantage of allowing the deepening and superficialization of anesthesia. It should, however, be administered with an intravenous agent or other better tolerated inhalation agents, such as halothane or sevoflurane ${ }^{27}$.

Low flow leads to lower consumption and pollution and better moistening of the inhalation system and airways; there must, however, be a permanent care to avoid hypoxic mixtures. This way, when the option is basal flow, which is the administration of oxygen flow similar to consumption plus an additional equal or slightly higher nitrous oxide flow, it is important to know that nitrogen build up, common with the use of low flow, will further dilute oxygen in the lungs ${ }^{28}$. It is mandatory the monitoring of $\mathrm{FiO}_{2}$ and $\mathrm{FEO}_{2}$.

In addition, if dehydrated soda lime is suspected, water should be put in the upper part of the reservoir $(5 \mathrm{ml}$ for each $100 \mathrm{ml}$ lime) to prevent the formation of carbon monoxide 20,21 .

\section{CONSUMPTION AND COSTS}

Consumption is a major cost factor. Desflurane is very volatile and has high MAC; so, its consumption should be de- creased for the cost to be affordable. One of the most important elements in the composition of inhalation agents consumption is fresh gas flow. So, techniques using low flow are very important for the administration of this agent.

\section{Observing the formula to calculate consumption:}

Consumption $=\mathrm{C} \times \mathrm{F} \times \mathrm{T} / \mathrm{D} \times \mathrm{Vm} \times \mathrm{AT}+\mathrm{t}^{\circ} \mathrm{C} / \mathrm{MW} \times 273$

Where:

$\mathrm{C}=$ concentration in $\%$ volume

$\mathrm{F}=$ flow in $\mathrm{ml} \cdot \mathrm{min}^{-1}$

$\mathrm{T}=$ time in minutes

$\mathrm{D}=$ Density

$\mathrm{Vm}=$ Molecular volume in liters $(22.4 \mathrm{I})$

AT $=$ absolute temperature $\left(273^{\circ}\right)$

$\mathrm{T}^{\circ} \mathrm{C}=$ room temperature in degrees $\mathrm{C}$

$\mathrm{MW}=$ molecular weight

It is seen that consumption is a direct function of flow, concentration and time, and an indirect function of density/molecular weight (MW) ratio ${ }^{4}$.

Table IV shows that with low flows ( 1 L. $\left.\mathrm{min}^{-1}\right)$ to maintain anesthesia after saturation of the inhalation system of the anesthesia machine, it is possible to obtain low consumption to result in affordable cost ${ }^{4}$. These data are confirmed by other authors $^{29,30}$.

Table IV - Consumption of Low Gas Flow Inhalation Anesthetics $^{4}$

\begin{tabular}{lc}
\hline Anesthetic Agent & $\begin{array}{c}\text { Mean Volume }(\mathrm{ml}) \\
\text { during } 20 \text { Initial Minutes }\end{array}$ \\
\hline Halothane & 8.16 \\
Isoflurane & 11.6 \\
Sevoflurane & 8.39 \\
Desflurane & 11.89 \\
\hline
\end{tabular}

\section{CONCLUSION}

Desflurane is a step further toward the ideal anesthetic agent. Its physicochemical properties give it well desirable pharmacokinetic characteristics, such as very fast induction (progression) and recovery, and minimal metabolization with very low organic toxicity and high molecular stability, even in the presence of carbon dioxide.

Its pharmacodynamic actions are perfectly tolerated and compatible with its clinical use.

If care is taken with vaporization, storage and consumption, desflurane may be even used in large scale, especially in outpatient anesthesia, severely ill patients, liver and renal disease patients and the elderly, and for long procedures. 


\section{REFERÊNCIAS - REFERENCES}

01. Eger II EI - New inhaled anesthetics. Anesthesiology, 1994;80:906-922.

02. Eger II El-Physicochemical properties and pharmacodynamics of desflurane. Anaesthesia, 1995;50:(Suppl):3-8.

03. Graham SC - The desflurane Tec 6 Vaporizer. Br J Anaesth, 1994;72:470-473.

04. Borges MMJ, Saraiva RA - Seqüência de fluxo de gás fresco para o início da anestesia com baixo fluxo. Aplicação clínica do estudo teórico de Mapleson. Rev Bras Anestesiol, 2002;52:146-155.

05. Eger II EI, Stability of I - 653 in sodalime. Anesth Analg, 1987;66:983-985.

06. Saraiva RA - Farmacocinética dos Anestésicos Inalatórios, em: Manica JM - Anestesia, Princípios e Técnicas. $3^{a} \mathrm{Ed}$, Porto Alegre, Artes Médicas, 2002;241-250.

07. Weiskopf RB - Cardiovascular effects of desflurane in experimental animals and volunteers. Anaesthesia, 1995;50:(Suppl): 14-17.

08. Ebert TJ, Arain SR - Renal responses to low flow desflurane, sevoflurane and propofol in patients. Anesthesiology, 2000;93:1401-1406.

09. James RM, Kollen DD, Cashman JN et al - Biotransformation and hepato-renal function in volunteers after exposure to desflurane (I - 653). Br J Anaesth, 1992;64:482-487.

10. Sutton TS, Koblin DD, Guienke KD et al - Fluoride metabolities following prolonged exposure of volunteers and patients to desflurane. Anesth Analg, 1991;73:180-185.

11. Costa VV, Saraiva RA - Ação do óxido nitroso no sistema nervoso central. Estudo eletroneurofisiológico como agente único e como agente coadjuvante. Rev Bras Anestesiol, 2002;52:255-271.

12. Rampil I, Lockhart S, Eger II El et al - The electroencephalographic effect of desflurane in humans. Anesthesiology, 1991; 74:434-439.

13. Ornstein E, Young W, Fleishcher $L$ et al - Desflurane and isoflurane have similar effects on cerebral blood flow in patients with intracranial mass lesions. Anesthesiology, 1993;79: 498-502.

14. Ghouri AF, Bodner M, White PF - Recovery profile after desflurane nitrous oxide versus isoflurane-nitrous oxide in outpatients. Anesthesiology, 1991;74:419-424.

15. Magalhães E - Avaliação da função tubular renal em profissionais expostos cronicamente a agentes anestésicos fluorados. Tese de Doutorado apresentado à Escola Paulista de Medicina. Universidade Federal de São Paulo. São Paulo, 1998.

16. Wissing MD, Kuhn I, Warnken $U$ et al - Carbon Monoxide production from desflurane, enflurane, halothane, isoflurane and sevoflurane with dry soda lime. Anesthesiology, 2001;95: 1205-1212.

17. Norkool DM, Kirkpalrick JN - Treatment of acute carbon monoxide poising with hyperbaric oxygen. A review of 115 cases. Ann Emerg Med, 1985;14:1168-1182.

18. Krantz T, Thisted B, Strom J et al - Acute carbon monoxide poisoning. Acta Anaesthesiol Scand, 1988;32:278-289.

19. Pace $n$, Stragman E, Walker E - Acceleration of carbon monoxide elimination by high pressure oxygen. Science, 1950;111: 652-664.

20. Fang ZX, Eger II EI, Laster MJ et al - Carbon monoxide production from degradation of desflurane, enflurane, isoflurane, halothane and sevoflurane by soda time and baralyme. Anesth Analg, 1995;80:1187-1193.

21. Strum D, Eger II EI - The degradation, absorption and solubility of volatile anesthetics in soda lime depend on water contend. Anesth Analg, 1994;78:340-348.
22. Newmann MA, Laster MJ, Weiskopt RB et al - The elimination of sodium and potassium hydroxides from desiccated soda lime diminishes degradation of desflurane to carbon monoxide and sevoflurane to compound a but does not compromise carbon dioxide absorption. Anesth Analg, 1999;89:768-773.

23. Weiskopf RB - Implications of chemical and physical properties of desflurane for longer surgery. Anaesthesia, 1995;50:(Suppl): 9-13.

24. Weiskopf RB - Cardiovascular effects of desflurane in experimental animals and volunteers. Anaesthesia, 1995;50:(Suppl): 14-17.

25 . Kortila K - Recovery from out patient anaesthesia. Anaesthesia, 1995;50:(Suppl):22-28.

26. Conzen $\mathrm{P}$, Peter $\mathrm{K}$ - Inhalation anaesthesia at the extremes of age: geriatric anaesthesia. Anaesthesia, 1955;50:(Suppl): 29-33.

27. Olsson GL - Inhalational anaesthesia at the extremes of age: paediatric anaesthesia. Anaesthesia, 1955;50:(Suppl):29-33.

28. Baum JA, Aitkenhead - Low-flow anaesthesia. Anaesthesia, 1995;50:(Suppl):37-44

29. Lockwood GC, White DC - Measuring the costs of inhaled anesthetics. Br J Anaesth, 2001; 87:559-563.

30. Eger II EI - Economic analysis and pharmaceutical policy: a consideration of the economics of the use of desflurane. Anaesthesia, 1995;50:(Suppl):45-48.

\section{RESUMEN}

Saraiva RA - Desflurano: Propiedades Físico-Químicas, Farmacologia y Uso Clínico

Justificativa y Objetivos - Siguiendo el desenvolvimiento de la química nuclear con la síntesis de los halogenados desde la década de 50 en el siglo pasado, varios agentes fueron ensayados clínicamente y algunos tuvieron grande aplicación práctica. La búsqueda por el agente ideal continua. Actualmente están en uso clínico el halotano, enflurano, isoflurano, sevoflurano y desflurano. Todos presentan ventajas y desventajas. El desflurano es el más reciente de estos agentes. El objetivo de este trabajo es describir las propiedades físico-químicas y farmacológicas del desflurano y relatar la aplicación clínica obtenida con el uso de este nuevo agente.

Contenido - Las propiedades físico-químicas y las características farmacocinéticas y farmacodinámicas son determinantes del uso clínico del desflurano. Teniendo punto de ebullición bajo, volatiliza fácilmente en las temperaturas de las salas de operación, y su CAM elevada requiere que sea administrado en concentraciones altas. Entonces, es recomendable el uso de flujo bajo de gas fresco y vaporizador especial para que su aplicabilidad clínica sea económicamente viable. Además de esto, el uso de un agente coadyuvante, como el óxido nitroso, reduce su CAM y posibilita que sea usado en menores concentraciones. Su farmacocinética permite inducción y regresión rápida, destacándose también que tiene molécula muy estable, siendo muy poco metabolizado, ofreciendo grande tolerabilidad para el organismo humano. Sus repercusiones farmacodinámicas son dosis dependientes, semejantes a los demás agentes inhalatorios potentes.

Conclusiones - El desflurano representa una etapa a más en la evolución para llegar al anestésico ideal. Sus propiedades físico-químicas le confieren características farmacocinéticas bastante deseables, que propician inducción (progresión) y regresión rápidas y también metabolización mínima con la más baja toxicidad orgánica entre los anestésicos halogenados, y fuerte estabilidad molecular, inclusive en la presencia de absorbentes de dióxido de carbono. Tomándose las debidas precauciones cuanto a la vaporización, almacenamiento y consumo, el desflurano puede ser usado inclusive en larga escala, siendo económicamente viable. 